\title{
Super-High-Frequency SAW Resonators on AlN/Diamond
}

\author{
J. G. Rodríguez-Madrid, G. F. Iriarte, J. Pedrós, O. A. Williams, D. Brink, and F. Calle
}

\begin{abstract}
This letter describes the procedure to manufacture high-performance surface acoustic wave (SAW) resonators on AIN/diamond heterostructures working at frequencies beyond $10 \mathrm{GHz}$. In the design of SAW devices on AIN/diamond systems, the thickness of the piezoelectric layer is a key parameter. The influence of the film thickness on the SAW device response has been studied. Optimized thin films combined with advanced e-beam lithographic techniques have allowed the fabrication of one-port SAW resonators with finger width and pitch of $200 \mathrm{~nm}$ operating in the 10-14 GHz range with up to $36 \mathrm{~dB}$ out-of-band rejection.
\end{abstract}

Index Terms-AIN/diamond, surface acoustic wave (SAW) resonator, super-high-frequency band, thickness influence.

\section{INTRODUCTION}

T HE INCREASING volume of information in data transmission systems results in a growing demand of applications working in the super-high-frequency band $(3-30 \mathrm{GHz})$. Also, in gas sensor applications, the sensitivity is proportional with the square of the resonance frequency. In order to increase the frequency, two approaches may be followed: 1) to improve lithography resolution and 2) to exploit confined modes in proper layer-substrate combinations (in particular, slow-on-fast structures). In this letter, both approaches have been done using the high sound velocity of some modes in AlN/diamond [1], [2] and submicrometer interdigital transducer (IDT) periods [3], [4]. The AIN/diamond system has attracted considerable interest recently not only for the combination of the strong piezoelectricity of AIN with the SAW velocity of diamond (of about $12000 \mathrm{~m} / \mathrm{s}$ [5], the largest of all materials) but also for its suitability for harsh environments [6]. In such a layered system, the electromechanical coupling coefficient $\left(K^{2}\right)$ and the propagation velocity $v$ of the acoustic waves depend on the film-thickness-to-wavelength ratio $(H / \lambda)$. At a small $H / \lambda$, the SAW extends far into the underlying solid, and $v$ approaches the large velocity value of the bare substrate, although $K^{2}$ is reduced due to the nonpiezoelectricity of diamond. Conversely, at a large $H / \lambda$, the SAW is mostly concentrated in the film region. Thus, $K^{2}$ is large, but $v$ is limited by the smaller velocity value of the AIN overlayer. In addition to the intrinsic limitations imposed by the dispersion of the material system, the crystal quality and orientation of the sputtered AIN thin films, and thus their piezoelectricity, are limited by the film thickness [7], [8]. Moreover, the insulating character of AlN makes the e-beam patterning of the submicrometer IDTs more difficult. Therefore, the design of high-frequency and low-loss AlN/diamond-based SAW devices requires careful selection of the film thickness, which should be a tradeoff among the different aspects mentioned earlier.

In this letter, we report on the influence of the film thickness on the response of super-high-frequency SAW devices fabricated on AlN/diamond systems using nanometer-scale IDTs. One-port SAW resonators working in the $10-14-\mathrm{GHz}$ regime with good out-of-band rejection have been developed on tailored AlN/diamond structures. These frequencies are the highest obtained for this material system and almost double the maximum value of $7.4 \mathrm{GHz}$ reported so far [5], [9], [10].

\section{EXPERIMENTAL}

AIN films with thickness ranging from $150 \mathrm{~nm}$ to $1.2 \mu \mathrm{m}$ were deposited in a home-built balanced magnetron sputter deposition system on polished microcrystalline diamond substrates. The specific parameters used for the deposition of very thin (0002)-textured AIN films are described elsewhere [7], [11]. Fifty-nanometer-thick Aluminium IDTs and reflecting gratings were fabricated by e-beam lithography. The nominal period and metallization ratio were $800 \mathrm{~nm}$ and 0.5 , respectively, i.e., finger width and pitch of $200 \mathrm{~nm}$. The number of finger pairs was 100 for both the IDT and the reflector, whose finger length was $6.2 \mu \mathrm{m}$. The dimensions of the nanometerscale patterns were confirmed by scanning electron microscopy (SEM) and atomic force microscopy (AFM) analysis, as depicted in Fig. 1.

\section{RESULTS AND DISCUSSION}

In order to study the influence of the piezoelectric film thickness on the SAW response, one-port SAW resonators with a period of $800 \mathrm{~nm}$ were manufactured using 150-, 300-, 600-, and 1200 -nm-thick AIN films on diamond $(k H=2 \pi H / \lambda=$ $1.18,2.36,4.71$, and 9.42 , respectively). 

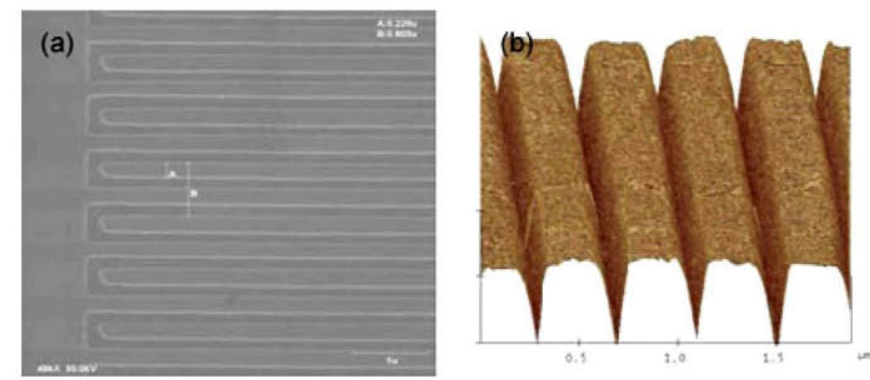

Fig. 1. (a) SEM and (b) AFM images of a $\lambda=800 \mathrm{~nm}$ IDT on AlN/diamond.

The quality of the AIN film, and hence its piezoelectricity, is thickness-dependent. The compromise between selecting the right thickness while maintaining the crystallographic quality of the piezoelectric film is evident with the experimental data presented in this letter. Thus, for an optimal design structure (IDT/piezoelectric film/diamond substrate), the $k H$ providing a mode with the best combination of $v$ and $K^{2}$ is selected. This choice implies a compromise between the frequency and the intensity of the targeted resonances, i.e., a larger frequency can be achieved at the cost of larger losses or vice versa due to the $K^{2}$ behavior discussed later. Once $k H$ is chosen and since $f=$ $v / \lambda$, the smallest $\lambda$ that can be reliably patterned is selected. This also requires a film thickness large enough to ensure a good crystallographic quality. Since, as shown elsewhere [11], this is a limiting factor, a compromise should be made again between the achievable frequency and intensity (loss) of the targeted resonances.

Rayleigh and Sezawa (confined) modes are presented in similar structures as the one presented here due to the higher $v$ of the substrate in comparison with the $v$ of the piezoelectric film [12], [13]. Fig. 2 shows the reflection spectra of the fabricated devices. For the SAW resonators on the 150-, 300-, and 600-nm-thick AIN films, the main resonance corresponds to the first-order Sezawa mode (labeled as $S_{1}$ ) at 14.0, 12.6, and $10.9 \mathrm{GHz}$, respectively. However, for the SAW device on the 1200 -nm-thick film, the main resonance (at $11.5 \mathrm{GHz}$ ) corresponds to the second Sezawa mode $\left(S_{2}\right)$. These frequencies are much higher than the largest value of $7.4 \mathrm{GHz}$ obtained for this material system so far [10]. In addition, the quality factor $Q$ and the out-of-band rejection for the $S_{1}$ mode in the 300 -nm-thick film are 12560 and $36.5 \mathrm{~dB}$, respectively. These values are among the largest reported for a SAW resonator [9].

The identification of the acoustic modes is based on the numerical calculation of the dispersion of their velocity using a method based on the Green's function formalism [14], which was developed to calculate surface Brillouin spectra. In addition, the theoretical values of $K^{2}$ were calculated using the transfer matrix approach [15]. The material parameters used are listed elsewhere [16]. Fig. 3 shows the calculated dispersion of the SAWs and pseudoSAWs propagating in the $\mathrm{AIN}(0001) /$ diamond(111) heterostructure. The spectrum of SAWs comprises the Rayleigh $(R)$ and the Sezawa $\left(S_{i}\right)$ modes that propagate with velocities below that of the transverse bulk wave in the substrate [12]. The gray scale represents the magnitude of the shear vertical component of the waves. In this scale, brighter areas indicate larger magnitude. The experimental data,

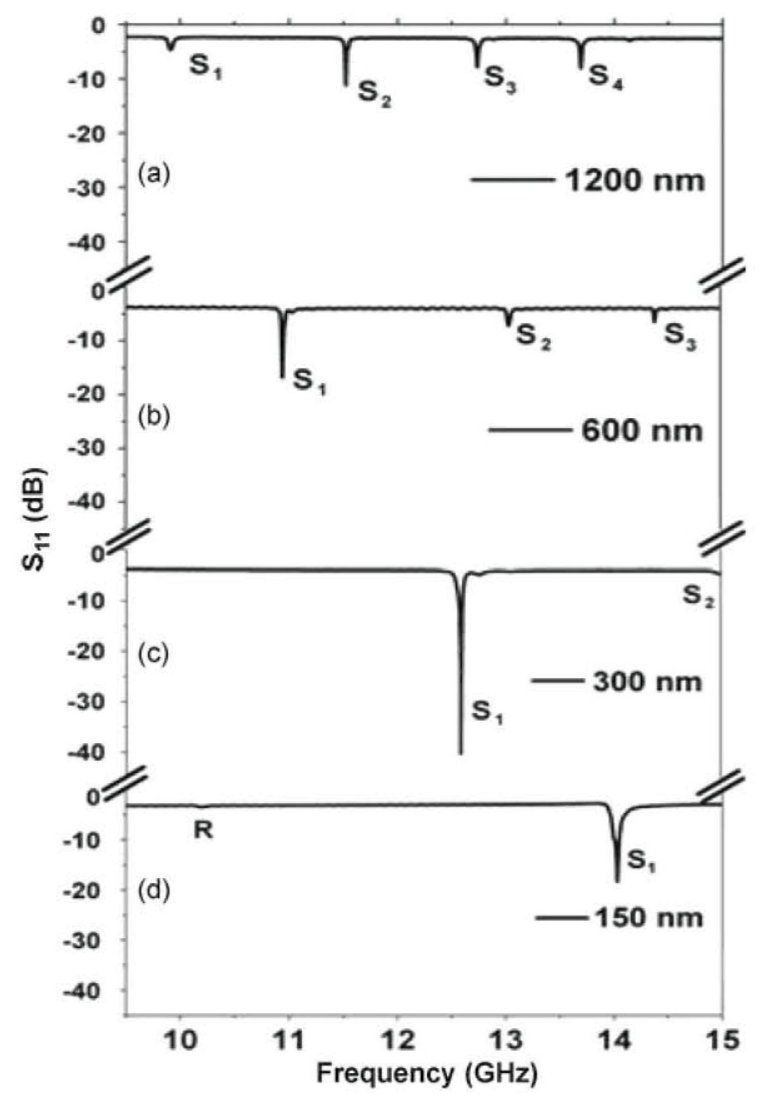

Fig. 2. Reflection coefficient $\left(S_{11}\right)$ spectra for identical $(\lambda=800 \mathrm{~nm})$ oneport SAW resonators on (a) 1200-, (b) 600-, (c) 300-, and (d) 150-nm-thick AlN films on diamond. $R$ and $S_{i}$ denote the Rayleigh and Sezawa modes, respectively.

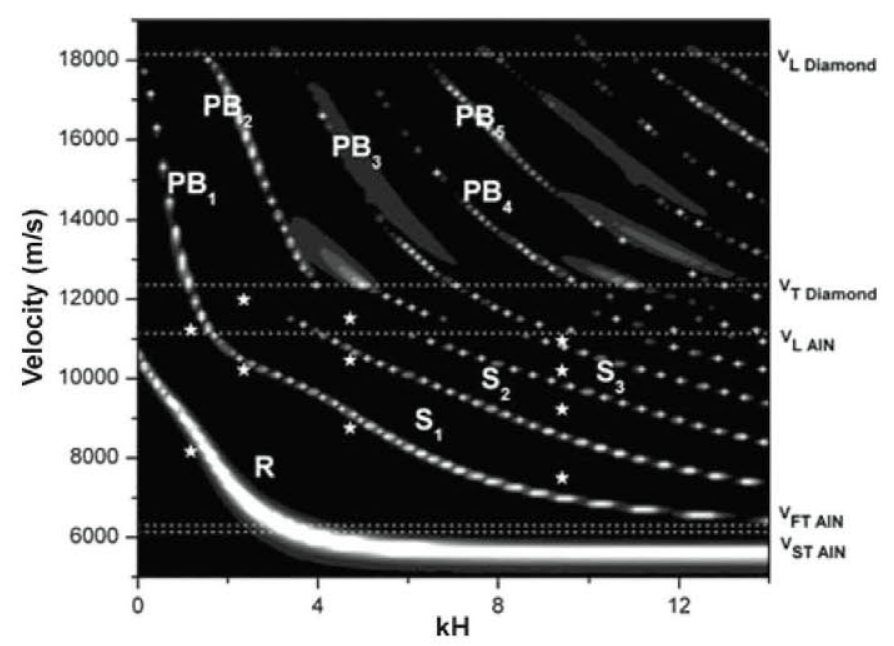

Fig. 3. Dispersion of the velocity of the Rayleigh $(R)$, Sezawa $\left(S_{i}\right)$, and pseudobulk $\left(P B_{i}\right)$ modes on AlN/diamond. The gray-scale traces correspond to the calculated modes, whereas the experimental data are represented by white solid stars.

obtained from the resonance frequencies in Fig. 2, are indicated by white solid stars.

The velocity of the modes decreases from $k H=0$ (diamond) to $k H=\infty$ (AlN) indicating that the thickness of the piezoelectric film needs to be reduced in order to obtain higher frequencies. However, the dispersion of $K^{2}$ is not a monotonic function of $k H$, as shown elsewhere [9], [17]. Fig. 4 shows the 


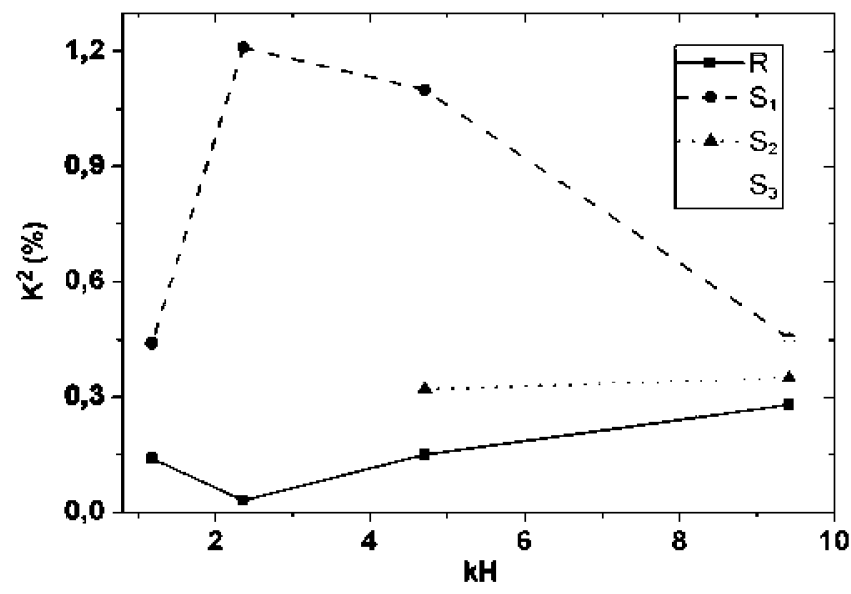

Fig. 4. Calculated electromechanical coupling coefficient $\left(K^{2}\right)$ for the Rayleigh $(R)$ and Sezawa $\left(S_{i}\right)$ modes as a function of the normalized piezoelectric film thickness on the AlN/diamond structure $(k H)$. The wavelength is $800 \mathrm{~nm}$ in all the cases.

calculated $K^{2}$ for the different $k H$ studied here. The best $K^{2}$ value for the $S_{1}$ mode (1.21\%) is obtained for the 300 -nm-thick AlN film $(k H=2.36)$, in agreement with previous calculations [9], [17]. On the other hand, from the point of view of the film quality, the degree of the $c$-axis orientation of the AIN film, and thus its piezoelectricity, decreases as its thickness is reduced [11]. According to our results and the results of [18] on sputtered AlN, the full-width at half-maximum (FWHM) of the (0002)-rocking curve must typically be smaller than $4^{\circ}$ in order to yield a strong piezoelectric response. Therefore, the 300 -nm-thick AlN film $(k H=2.36)$ corresponds to the optimum case, where a very high frequency is achieved while keeping a maximum $K^{2}$ for the $S_{1}$ mode and an FWHM of the AlN layer just below $4^{\circ}[11]$, both conditions ensuring low loss.

\section{Conclusion}

AlN films of different thickness have been deposited by reactive sputtering on diamond substrates in order to fabricate tailored SAW resonators for high-frequency applications. We have demonstrated that for very-high-frequency $(12-14 \mathrm{GHz})$ and low-loss SAW devices, the film thickness should be kept well below the SAW wavelength $(k H=1.2-2.4)$ but has to be large enough to ensure a rocking-curve FWHM value of the $\mathrm{AlN}(0002)$ reflection of at least $4^{\circ}$ as measured by X-ray diffraction. In particular, for a $\lambda$ of $800 \mathrm{~nm}$, the optimum AIN layer thickness is $300 \mathrm{~nm}$. This structure provides an $S_{1}$ mode operating at $12.6 \mathrm{GHz}$ with a $Q$ factor of 12560 and an out-ofband rejection of $36.5 \mathrm{~dB}$. The frequency of this mode has been shifted to $14.0 \mathrm{GHz}$ using a 150 -nm-thick AlN film, although the reduced crystal quality and $K^{2}$ yield lower $Q$ factor and out-of-band rejection values.

\section{REFERENCES}

[1] H. Nakahata, K. Higaki, S. Fujii, A. Hachigo, H. Kitabayashi, K. Tanabe, Y. Seki, and S. Shikata, "SAW devices on diamond" in Proc. IEEE Ultrason. Symp., 1995, vol. 1, pp. 361-370.

[2] C. M. Flannery, M. D. Whitfield, and R. B. Jackman, "Surface acoustic wave properties of free-standing diamond films," Trans. Ultrason., Ferroelectr, Freq. Control, vol. 51, no. 3, pp. 368-371, Mar. 2004.

[3] T. Palacios, F. Calle, E. Monroy, J. Grajal, M. Eickhoff, O. Ambacher, and C. Prieto, "Nanotechnology for SAW devices on AlN epilayers," Mater. Sci. Eng. B, vol. 93, no. 1-3, pp. 154-158, May 2002.

[4] A. Müller, D. Neculoiu, G. Konstantinidis, G. Deligeorgis, A. Dinescu, A. Stavrinidis, A. Cismaru, M. Dragoman, and A. Stefanescu, "SAW devices manufactured on GaN/Si for frequencies beyond $5 \mathrm{GHz}$," IEEE Electron Device Lett., vol. 31, no. 12, pp. 1398-1400, Dec. 2010.

[5] P. Kirsch, M. B. Assouar, V. Mortet, P. Alnot, and O. Elmazria, "5 GHz surface acoustic wave devices based on aluminum nitride/diamond layered structure realized using electron beam lithography," Appl. Phys. Lett., vol. 88, no. 22, pp. 223 504-1-223 504-3, May 2006.

[6] M. R. Werner and W. R. Fahrner, "Review on materials, microsensors, systems, and devices for high-temperature and harsh-enviroment applications," IEEE Trans. Ind. Electron., vol. 48, no. 2, pp. 249-257, Apr. 2001.

[7] G. F. Iriarte, J. G. Rodríguez, and F. Calle, "Synthesis of $c$-axis oriented AlN thin films on different substrates: A review," Mater. Res. Bull., vol. 45 , no. 9 , pp. 1039-1045, Sep. 2010.

[8] R. S. Naik, J. J. Lutsky, R. Reif, C. G. Sodini, A. Becker, L. Fetter, H. Huggins, R. Miller, J. Pastalan, G. Rittenhouse, and Y.-H. Wong, "Measurements of the bulk, c-axis electromechanical coupling constant as a function of AlN film quality," Trans. Ultrason., Ferroelectr, Freq. Control, vol. 47, no. 1, pp. 292-296, Jan. 2000.

[9] M. Benetti, D. Cannatà, F. di Pietrantonio, and E. Verona, "Growth of AlN piezoelectric film on diamond for high-frequency surface acoustic wave devices," Trans. Ultrason., Ferroelectr., Freq. Control, vol. 52, no. 10, pp. 1806-1811, Oct. 2005.

[10] P. Kirsch, M. B. Assouar, O. Elmazria, M. E. Hakiki, V. Mortet, and P. Alnot, "Combination of e-beam lithography and of high velocity AIN/diamond-layered structure for SAW filters in X band," Trans. Ultrason., Ferroelectr, Freq. Control, vol. 54, no. 7, pp. 1486-1491, Jul. 2007.

[11] J. G. Rodríguez-Madrid, G. F. Iriarte, D. Araujo, M. P. Villar, O. A. Williams, W. Müller-Sebert, and F. Calle, "Optimization of AIN thin layers on diamond substrates for high frequency SAW resonators," Mater. Lett., vol. 66, no. 1, pp. 339-342, Jan. 2012.

[12] J. Pedrós, F. Calle, J. Grajal, R. J. Jiménez Riobóo, Y. Takagaki, K. H. Ploog, and Z. Bougrioua, "Anisotropy-induced polarization mixture of surface acoustic waves in GaN/c-sapphire heterostructures," Phys. Rev. B, vol. 72, no. 7, pp. 075306-1-075306-7, Aug. 2005.

[13] Y. Takagaki, T. Hesjedal, O. Brandt, and K. H. Ploog, "Surface-acousticwave transducers for the extremely-high-frequency range using $\mathrm{AlN} / \mathrm{SiC}$ (0001)," Semicond. Sci. Technol., vol. 19, no. 2, pp. 256-259, Feb. 2004.

[14] A. G. Every, "Measurement of the near-surface elastic properties of solids and thin supported films," Meas. Sci. Technol., vol. 13, no. 5, p. R21, May 2002.

[15] A. H. Fahmy and E. L. Adler, "Propagation of acoustic surface wave in multilayers: A matrix description," Appl. Phys. Lett., vol. 22, no. 10, pp. 495-497, May 1973.

[16] M. Benetti, D. Cannata, F. Di Pietrantonio, V. I. Fedosov, and E. Verona, "Gigahertz-range electro-acoustic devices based on pseudo-surfaceacoustic waves in AlN/diamond/Si structures," Appl. Phys. Lett., vol. 87, no. 3, pp. 033504-1-033504-3, Jul. 2005.

[17] G. F. Iriarte, "Surface acoustic wave propagation characteristics of aluminum nitride thin films grown on polycrystalline diamond," $J$. Appl. Phys., vol. 93, no. 12, pp. 9604-9609, Jun. 2003.

[18] K. Tonisch, V. Cimalla, C. Foerster, H. Romanus, O. Ambacher, and D. Dontsov, "Piezoelectric properties of polycrystalline AIN thin films for MEMS application," Sens. Actuators A, Phys., vol. 132, no. 2, pp. 658663, Nov. 2006. 